\title{
Luigi Maiuri: un Grande Uomo - a Great Spirit
}

Mauro Piacentini ${ }^{1,2}$ and Guido Kroemer ${ }^{3,4,5,6,7}$

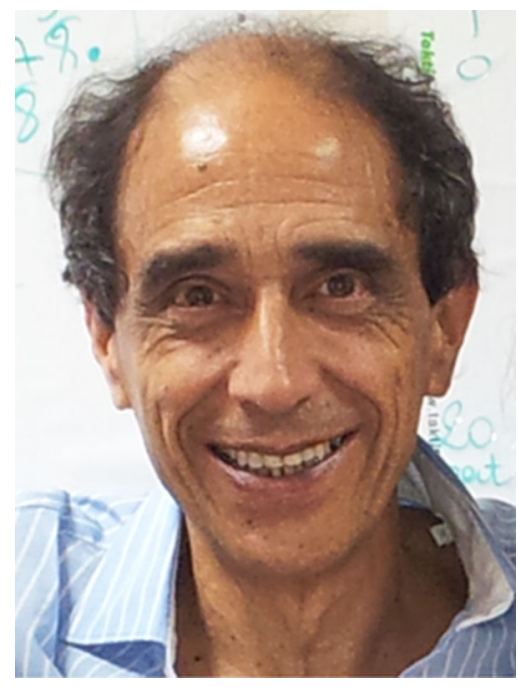

On the frontispiece of the Parisian Pantheon, the following inscription is engraved: "AUX GRANDS HOMMES, LA PATRIE RECONNAISSANTE" ("To the Great Men, the Grateful Homeland"). Logically, one of the hotels close to the Pantheon is called "Aux Grands Hommes" (always with capital letters), where Luigi stayed usually when he came to Paris. Today the inscription on the Pantheon has become "politically incorrect", because it is not any more acceptable that humanity is only represented by men and because "homeland" has the odour of rancid patriotism. It is not any more conceivable that a country aspires at representing the entire universe as this was in the case during the French Revolution. The translation of the sense of the inscription to contemporary

Correspondence: Mauro Piacentini (mauro.piacentini@uniroma2.it)

'Department of Biology, University of Rome 'Tor Vergata', Rome, Italy

${ }^{2}$ National Institute for Infectious Diseases IRCCS 'L. Spallanzani', Rome, Italy

Full list of author information is available at the end of the article.
English might be something like "To the great spirits, the grateful universe".

Luigi was rather small in stature and thin in complexion, nervous in his gesticulation and baroque in his expression, and he was a "Grand Homme" (in Italian "Grande Uomo"), a Great Spirit, a scientist and paediatrician with exquisite manners, irradiating an exceptional kindness, complete dedication to translational research, and an incisive intellectual curiosity.

Luigi (born in 1954, in Cosenza, Southern Italy), studied medicine in Rome, became a paediatrician in Naples, transited the University of Foggia, worked as honorary lecturer and professor at University College London and University of Southampton, and finally was affiliated to the University of Piemonte Orientale and the San Raffaele Hospital in Milan, where he was directing the European Institute for Research in Cystic Fibrosis.

Luigi's research was always dedicated to the exploration of the molecular mechanism of human pathologies. $\mathrm{He}$ started his own research group relatively late, and his team was the first to identify the importance of innate immune responses and interleukin-15 for the pathogenesis of celiac disease ${ }^{1}$ and to develop mouse models of autoimmune thyroiditis ${ }^{2}$.

Luigi spent most of his carrier on cystic fibrosis, which is the most frequent monogenetic lethal disease in humans. He discovered that cystic fibrosis is linked to a defect in autophagy in respiratory epithelial cells and other cell types ${ }^{3-5}$. This important discovery allowed him to develop novel combination therapies involving autophagy inducers, including the combination of cysteamine and epigallocatechine gallate. Using mouse models of cystic fibrosis, he showed that both agents act on target to improve cystic fibrosis by inhibiting transglutaminase-2 and by stimulating autophagy, thus engaging in a synergistic interaction ${ }^{6}$. In two independent clinical phase II trials, Luigi demonstrated that the combination of 
cysteamine and epigallocatechine gallate is active in children bearing the most frequent pathogenic mutation in the gene coding for cystic fibrosis transmembrane conductance regulator (CFTRdel508) ${ }^{7-10}$. He developed and proved the concept that drugs used for the treatment of cystic fibrosis can be tested on freshly brushed nasal epithelial cells and that such in vitro test accurately predict the in vivo response in the clinics ${ }^{11,12}$. He also pioneered the idea that the clinical efficacy of drugs should be assessed by assessing surrogate markers, such as measuring the functionality of CFTR ex vivo ${ }^{13,14}$. Within the realm of cell biology, Luigi developed the concept that cystic fibrosis causes a pro-inflammatory stress response that should be targeted by modulators of proteostasis including autophagy inducers, which thus would constitute an etiological treatment ${ }^{15-17}$.

Recently, Luigi discovered with his dedicated team of collaborators including his wife Valeria Raia that CFTR is not only involved in the pathogenesis of cystic fibrosis, the condition in which CFTR is inactivated due to loss-offunction mutations. Indeed, Luigi found that CFTR expressed on the surface of intestinal epithelial cells can be inhibited by a specific peptide from gliadin, a gluten compound, thus explaining the pathogenesis of celiac disease. A particular non-digested peptide from gliadin binds to CFTR, thus causing its inhibition in enterocytes, resulting in autophagy inhibition, transglutaminase- 2 activation, and pro-inflammatory stress ${ }^{18-20}$. This molecular cascade then lays the ground for stimulating an immune response against another gliadin-derived peptide fragment, resulting in a full-blown (auto-)immune response. Importantly, so called CFTR potentiators, i.e. agents that activate the chloride channel function of CFTR, can reduce the gliadin-mediated inhibition of CFTR, thus interrupting the pathogenic cascade at its apex and preventing the development of celiac disease in suitable mouse models ${ }^{19,20}$.

Luigi's fertile carrier was truncated on 9 February 2019. As his memory will persist in his family, friends, alumni, colleagues, and patients, Luigi will survive as a Great Spirit in the Pantheon of Science and Medicine.

\footnotetext{
Author details

'Department of Biology, University of Rome 'Tor Vergata', Rome, Italy. ${ }^{2}$ National Institute for Infectious Diseases IRCCS 'L. Spallanzani', Rome, Italy. ${ }^{3}$ Equipe labellisée par la Ligue contre le cancer, Université Paris Descartes, Université Sorbonne Paris Cité, Université Paris Diderot, Université Sorbonne Université, INSERM U1138, Centre de Recherche des Cordeliers, Paris, France.

${ }^{4}$ Metabolomics and Cell Biology Platforms, Institut Gustave Roussy, Villejuif,

France. ${ }^{5}$ Pôle de Biologie, Hôpital Européen Georges Pompidou, AP-HP, Paris, France. ${ }^{6}$ Suzhou Institute for Systems Biology, Chinese Academy of Sciences, Suzhou, China. 'Karolinska Institute, Department of Women's and Children's Health, Karolinska University Hospital, Stockholm, Sweden
}

Conflict of interest

The authors declare that they have no conflict of interest.

\section{Publisher's note}

Springer Nature remains neutral with regard to jurisdictional claims in published maps and institutional affiliations.

Received: 18 February 2019 Accepted: 19 February 2019

Published online: 01 March 2019

\section{References}

1. Maiuri, L. et al. Association between innate response to gliadin and activation of pathogenic T cells in coeliac disease. Lancet 362, 30-37 (2003).

2. Quaratino, S. et al. Degenerate self-reactive human T-cell receptor causes spontaneous autoimmune disease in mice. Nat. Med. 10, 920-926 (2004).

3. Luciani, A. et al. Cystic fibrosis: a disorder with defective autophagy. Autophagy 7, 104-106 (2011)

4. Luciani, A. et al. Defective CFTR induces aggresome formation and lung inflammation in cystic fibrosis through ROS-mediated autophagy inhibition. Nat. Cell Biol. 12, 863-875 (2010).

5. Luciani, A. et al. Targeting autophagy as a novel strategy for facilitating the therapeutic action of potentiators on DeltaF508 cystic fibrosis transmembrane conductance regulator. Autophagy 8, 1657-1672 (2012).

6. Ferrari, E. et al. Cysteamine re-establishes the clearance of Pseudomonas aeruginosa by macrophages bearing the cystic fibrosis-relevant F508del-CFTR mutation. Cell Death Dis. 8, e2544 (2017).

7. De Stefano, D. et al. Restoration of CFTR function in patients with cystic fibrosis carrying the F508del-CFTR mutation. Autophagy 10, 2053-2074 (2014).

8. Villella, V. R. et al. Towards a rational combination therapy of cystic fibrosis: How cystamine restores the stability of mutant CFTR. Autophagy 9, 1431-1434 (2013).

9. Villella, V. R. et al. Disease-relevant proteostasis regulation of cystic fibrosis transmembrane conductance regulator. Cell Death Differ. 20, 1101-1115 (2013).

10. IZZO, V. et al. Metabolic interactions between cysteamine and epigallocatechin gallate. Cell Cycle 16, 271-279 (2017).

11. Villella, V. R. et al. Targeting the intracellular environment in cystic fibrosis: restoring autophagy as a novel strategy to circumvent the CFTR defect. Front Pharmacol. 4, 1 (2013).

12. Tosco, A. et al. A novel treatment of cystic fibrosis acting on-target: cysteamine plus epigallocatechin gallate for the autophagy-dependent rescue of class IImutated CFTR. Cell Death Differ. 23, 1380-1393 (2016).

13. Maiuri, L. \& Kroemer, G. Autophagy delays progression of the two most frequent human monogenetic lethal diseases: cystic fibrosis and Wilson disease. Aging 10, 3657-3661 (2018).

14. Tosco, A. et al. A novel treatment of cystic fibrosis acting on-target: cysteamine plus epigallocatechin gallate for the autophagy-dependent rescue of class IImutated CFTR. Cell Death Differ. 24, 1305 (2017).

15. Maiuri, L., Raia, V. \& Kroemer, G. Strategies for the etiological therapy of cystic fibrosis. Cell Death Differ. 24, 1825-1844 (2017).

16. Romani, L. et al. Thymosin alpha1 represents a potential potent singlemolecule-based therapy for cystic fibrosis. Nat. Med. 23, 590-600 (2017).

17. Zhang, S. et al. Evaluation of autophagy inducers in epithelial cells carrying the DeltaF508 mutation of the cystic fibrosis transmembrane conductance regulator CFTR. Cell Death Dis. 9, 191 (2018).

18. Luciani, A. et al. Lysosomal accumulation of gliadin p31-43 peptide induces oxidative stress and tissue transglutaminase-mediated PPARgamma downregulation in intestinal epithelial cells and coeliac mucosa. Gut 59, 311-319 (2010).

19. Villella, V. R. et al. A pathogenic role for cystic fibrosis transmembrane conductance regulator in celiac disease. EMBO J. 38, e100101 (2019).

20. Maiuri, L., Villella, V. R., Piacentini, M., Raia, V. \& Kroemer, G. Defective proteostasis in celiac disease as a new therapeutic target. Cell Death Dis. 10, 114 (2019). 